\title{
BR-dependent phosphorylation modulates PIF4 transcriptional activity and shapes diurnal hypocotyl growth
}

\author{
Stella Bernardo-García, ${ }^{1}$ Miguel de Lucas, ${ }^{1}$ Cristina Martínez, Ana Espinosa-Ruiz, \\ Jean-Michel Davière, and Salomé Prat \\ Centro Nacional de Biotecnología-Consejo Superior de Investigaciones Científicas, 28049 Madrid, Spain
}

\begin{abstract}
Signaling by the hormones brassinosteroid (BR) and gibberellin (GA) is critical to normal plant growth and development and is required for hypocotyl elongation in response to dark and elevated temperatures. Active $B R$ signaling is essential for GA promotion of hypocotyl growth and suppresses the dwarf phenotype of GA mutants. Cross-talk between these hormones occurs downstream from the DELLAs, as GA-induced destabilization of these GA signaling repressors is not affected by BRs. Here we show that the light-regulated PIF4 (phytochromeinteracting factor 4) factor is a phosphorylation target of the BR signaling kinase BRASSINOSTEROIDINSENSITIVE 2 (BIN2), which marks this transcriptional regulator for proteasome degradation. Expression of a mutated PIF41A protein lacking a conserved BIN2 phosphorylation consensus causes a severe elongated phenotype and strongly up-regulated expression of the gene targets. However, PIF41A is not able to suppress the dwarf phenotype of the bin2-1 mutant with constitutive activation of this kinase. PIFs were shown to be required for the constitutive BR response of bes1-D and bzr1-1D mutants, these factors acting in an interdependent manner to promote cell elongation. Here, we show that bes1-D seedlings are still repressed by the inhibitor BRZ in the light and that expression of the nonphosphorylatable PIF41A protein makes this mutant fully insensitive to brassinazole (BRZ). PIF41A is preferentially stabilized at dawn, coinciding with the diurnal time of maximal growth. These results uncover a main role of BRs in antagonizing light signaling by inhibiting BIN2-mediated destabilization of the PIF4 factor. This regulation plays a prevalent role in timing hypocotyl elongation to late night, before light activation of phytochrome B (PHYB) and accumulation of DELLAs restricts PIF4 transcriptional activity.
\end{abstract}

[Keywords: brassinosteroid; gibberellin; signal integration; plant growth]

Supplemental material is available for this article.

Received April 15, 2014; revised version accepted June 25, 2014.

De-etiolation involves a drastic change in plant morphology as germinating seedlings shift from dark to the light. In darkness, hypocotyl growth is rapidly induced at the expense of the cotyledons that remain folded, whereas the upper part of the hypocotyl forms an apical hook that protects the shoot apical meristem during rapid elongation in soil. Light arrests hypocotyl elongation and promotes opening and expansion of the cotyledons at the time that induces the differentiation of chloroplasts to start photosynthetic life. This photomorphogenic response is mediated through the activation of different families of photoreceptors, of which the red/far-red-absorbing phytochromes (PHYs) are the best characterized (Quail 2010). $\mathrm{PHYs}$ regulate light responses by inhibiting the PIFs (PHYinteracting factors), a family of basic helix-loop-helix

\footnotetext{
${ }^{1}$ These authors contributed equally to this work.

Corresponding author: sprat@enb.csic.es

Article is online at http://www.genesdev.org/cgi/doi/10.1101/gad.243675.114.
}

(bHLH) transcriptional regulators that promote etiolated growth in the dark (Leivar and Quail 2011). Although initially regarded as negative regulators of PHYB signaling, these factors are now recognized to function as main regulators of cell elongation and shade avoidance responses (Castillon et al. 2007; Nozue et al. 2007; De Lucas et al. 2008; Feng et al. 2008; Oh et al. 2009) by binding to G-box elements (CACGTG) and directly activate the expression of a large number of genes (Hornitschek et al. 2012; Zhang et al. 2013). Interaction with the Pfr form of PHYs induces phosphorylation of PIFs and marks these factors for proteolytic degradation, thus reversing PIF

(C) 2014 Bernardo-García et al. This article is distributed exclusively by Cold Spring Harbor Laboratory Press for the first six months after the fullissue publication date (see http://genesdev.cshlp.org/site/misc/terms. xhtml). After six months, it is available under a Creative Commons License (Attribution-NonCommercial 4.0 International), as described at http://creativecommons.org/licenses/by-nc/4.0/. 
transcriptional activity and promoting light-regulated gene expression (Shen et al. 2007; Lorrain et al. 2008). Consistent with this model of action, pif1pif3pif4pif5 quadruple mutants $(p i f q)$ display a constitutive photomorphogenic response in the dark and ectopic expression of chloroplast-related genes (Leivar et al. 2008).

Isolation of mutants with a de-etiolated response in the dark has also evidenced a role of the plant hormones gibberellin (GA) and brassinosteroid (BR) in suppressing light signaling. Mutants impaired in biosynthesis or signaling of these hormones have a characteristic dwarf phenotype and, in darkness, display short hypocotyls, an open apical hook, and activated expression of light-regulated genes as if they had received light (Alabadí et al. 2004; Nemhauser and Chory 2004). Loss-of-function mutations in the DELLA RGA and GAI genes suppress the de-etiolated phenotype of ga1-3 seedlings, thus demonstrating that constitutive photomorphogenesis in these GA-deficient mutants is caused by the accumulation of DELLAs (Alabadí et al. 2004). This family of nuclear repressors plays a central role in inhibiting GA-regulated gene expression (Fleet and Sun 2004) and is destabilized in the dark (Achard et al. 2007). GA sensing by the cytosolic receptor GID1 changes the receptor conformation and promotes the interaction of the GA-GID1 complex with the DELLAs (Murase et al. 2008; Shimada et al. 2008). Formation of a GA-GID1-DELLA complex favors ubiquitination of these repressors by the E3 ligase $\mathrm{SCF}^{\mathrm{SLY}}$ (Dill et al. 2004; Fu et al. 2004) and marks them for proteasomal degradation, allowing GA-induced hypocotyl growth. Work by our group and other groups has established that DELLAs bind the bHLH DNA recognition domain of PIFs, sequestering these factors into an inactive complex unable to bind to DNA (De Lucas et al. 2008; Feng et al. 2008; Gallego-Bartolomé et al. 2010). Nuclear balance of PIFs and DELLAs is therefore crucial to sustain the etiolated state of dark-grown seedlings and light-mediated degradation of PIFs together with the stabilization of DELLAs, providing a rapid mechanism to repress transcriptional activity of these factors during photomorphogenesis.

Etiolated growth also involves a prominent role of BR signaling, since BR synthesis and response mutants display a stronger de-etiolated phenotype in the dark than GA mutants (Clouse 2011). BR application rescues the germination phenotype of GA-insensitive mutants (Steber and McCourt 2001) and has a synergistic effect with GAs on hypocotyl elongation (Tanaka et al. 2003), suggestive of a cross-talk interaction of these hormones. In Arabidopsis, the BR signal transduction cascade is by now well understood, thanks to extensive molecular and genetic studies that contributed to the identification of all major signaling components in the pathway (Wang et al. 2012). BR's perception by the membrane receptor kinase BRASSINOSTEROID-INSENSITIVE1 (BRI1) (Kinoshita et al. 2005; She et al. 2011; Santiago et al. 2013) promotes association of this receptor kinase with the coreceptor BRI1-ASSOCIATED KINASE 1 (BAK1) (Nam and Li 2002; Wang et al. 2008) and induces a phosphorylation cascade that leads to activation of BRI1 SUPPRESSOR 1 (BSU1) (Mora-Garcia et al. 2004; Kim et al. 2009). This PP1-type phosphatase dephosphorylates the GSK3-like kinase BRASSINOSTEROID-INSENSITIVE 2 (BIN2) and inactivates this enzyme (Li and Nam 2002; Kim and Wang 2010). BIN2 has a negative regulatory function in BR signaling by phosphorylating two homologous transcription factors, BRI1-EMS SUPPRESSOR 1 (BES1) and BRASSINAZOLE-RESISTANT 1 (BZR1), to inhibit their transcriptional activity (He et al. 2002; Yin et al. 2002). When BR levels are elevated, BIN2 is inactivated, and BES1 and BZR1 are dephosphorylated by PP2A (Tang et al. 2011), the nonphosphorylated forms of these factors being shown to be more stable and to accumulate in the nucleus, where they bind the promoters of multiple genes and regulate their expression in a BR-dependent fashion (He et al. 2005; Yin et al. 2005; Sun et al. 2010).

Although BZR1 was originally identified as a repressor of BR synthesis (He et al. 2002; Wang et al. 2002) and BES1 was reported to heterodimerize with the BIM1 factor to activate gene expression (Yin et al. 2002, 2005), more recent studies showed that these factors bind similar BRRE and E-box elements and act as both activators and repressors, depending on the gene target (Sun et al. 2010; Yu et al. 2011). BZR1 has been recently shown to bind PIF4 and function as a PIF4 coactivator, with the BZR1-PIF4 complex binding a G-box motif identical to that bound by the PIF4 factor and synergistically activating a common set of genes (Oh et al. 2012). BES1 and BZR1 also interact with the DELLAs, these repressors blocking BES1 and BZR1 DNA-binding activity in a manner similar to that reported for PIF4 (Bai et al. 2012; Gallego-Bartolomé et al. 2012; Li et al. 2012). Interestingly, BZR1-dependent GAregulated genes largely overlap with the BZR1-PIF4 coactivated genes (Bai et al. 2012; Oh et al. 2012), suggesting that DELLAs' interaction with the BES1/BZR1 phosphorylation domain may compete for BZR1-PIF4 complex formation. Here, we show that the GSK3-like kinase BIN2, besides inactivating the BES1/BZR1 factors, phosphorylates PIF4/PIF5 to mark these transcription factors for proteasome degradation. This regulation is especially relevant late in the night, when PIF4 transcription is upregulated and the protein is stable. Although BES1 and BZR1 were reported to not be significantly affected by light (Luo et al. 2010), the active nonphosphorylated forms of the PIF4 and BES1/BZR1 factors overlap only during late night, BES1/BZR1 coactivator function thus having a relevant role in timing PIF4 transcriptional activity and in rhythmic hypocotyl growth. These results confirm a main function of the PIFs and BES1/BZR1 factors in cell elongation and highlight a role of these two families of bHLH factors as main integrators of light, BR, and GA signals by means of PHYB- and BIN2-induced destabilization and inactive complex formation with the DELLAs.

\section{Results}

Active BR signaling is required for GA promotion of hypocotyl growth

GA-insensitive sly1-10 and gai1 mutants exhibit seed germination defects, a phenotype that is alleviated by treatment with brassinolide (BL) (Steber and McCourt 
2001). Remarkably, we observed that BL application also rescues the short hypocotyl phenotype of these seedlings, suggesting that BR signaling overcomes the growthrepressing effects of DELLAs. Likewise, application of BL restores growth of ga1-3 GA-deficient seedlings and that of plants treated with the GA synthesis inhibitor paclobutrazol (PAC) (Supplemental Fig. S1). However, GAs are ineffective in promoting growth of det2-1 seedlings, deficient in BRs (Li et al. 1996), or that of wild-type seedlings treated with the BR synthesis inhibitor brassinazole (BRZ) (Nagata et al. 2000). These results are similar to those recently reported by Bai et al. (2012) and indicate that active $\mathrm{BR}$ signaling is required for the growth-promoting effects of GAs (Fig. 1A).

To investigate at which level of the GA signaling cascade BL exerts this cross-regulation, we examined whether BL application affects accumulation of the GA receptor GID1a by using GID1a-GFP transgenic lines (Willige et al. 2007). We also generated $\mathrm{p} R G A$ : :GFP-RGA det2.1 lines to test whether destabilization of this repressor is compromised in the BR-deficient background. As shown in Supplemental Figure S2A, neither hormone nor inhibitor treatments modified the levels of the GID1aGFP protein, indicating that BR signaling does not affect GID1 stability. Moreover, destabilization of the GFPRGA protein was similar in det2-1 lines to that in the wild-type controls (Fig. 1B), demonstrating that BRs do not affect GA-induced degradation of this repressor. Identical results were obtained by Western blot detection with an antibody raised against the RGA protein, thus excluding an anomalous response of the GFP-RGA fusion construct (Supplemental Fig. S2B). Destabilization of DELLAs in the det2-1 background, however, does not restore hypocotyl growth (Fig. 1B), supporting a regulatory role of BR signaling downstream from the DELLAs. Consistent with these results, we found that BRZ represses growth of the constitutive GA response tetra and global mutants, with knockout mutations in four or all five DELLA genes. These mutants show a dwarf phenotype when grown on BRZ (Supplemental Fig. S3), pointing to a role of a signaling component downstream from the DELLAs in GA and BR signaling cross-talk.

\section{BRs promote accumulation of a faster-migrating form of the PIF4 factor}

DELLAs repress growth by binding the bHLH DNA recognition domain of PIFs, which blocks the DNAbinding ability of these factors (De Lucas et al. 2008; Feng et al. 2008). The finding that BL promotes growth of mutants overaccumulating DELLAs and that these repressors are destabilized in det2-1 plants suggested that BRs may act at the level of PIFs. To test this hypothesis, we treated transgenic 35S: PIF4-HA plants with BL or BRZ to assess for changes in PIF4 protein levels. As shown in Figure 1C, a rapid accumulation of the PIF4HA protein was in fact observed in dark-grown seedlings after the application of $\mathrm{BL}$, indicating that stabilization of this factor is a primary response to BRs. Consistent with this observation, BRZ treatment reduced PIF4 levels, and this effect was abolished by pretreatment with the proteasome inhibitor MG132. Stabilization of the PIF4HA protein correlates also with a shift to a faster electrophoretic mobility band that may correspond to a nonphosphorylated form of this factor (Fig. 1C). Indeed, when plant extracts of mock-, BL-, and BRZ-treated seedlings were incubated with calf intestinal phosphatase (CIP), only the fastest PIF4-HA mobility band was observed (Fig. 1C), demonstrating that the slower-migrating forms correspond to phosphorylated isoforms of the protein. Therefore, these findings are suggestive of a role of BRs in PIF4 stabilization by inhibiting a protein kinase that tags this factor for proteasomal degradation.

PIF4-OE lines show a hyposensitive response to $B R Z$ and activated levels of expression of BR-regulated genes

If BRs act through the PIFs, higher levels of PIF4 activity should phenocopy BL effects. Indeed, PIF4-OE seedlings grown on BRZ are taller than wild-type controls, and these plants show a saturated response to BL (Supplemental Fig. S4). pif4pif5 seedlings, in contrast, display a hyposensitive response to this hormone and increased sensitivity to the inhibitor BRZ. An altered response toward BRZ was also observed after combined application of GA and BRZ. Pretreatment with this inhibitor blocks GA-induced elongation of wild-type and det2-1 seedlings but not that of the PIF4-OE or phyB mutant lines, which accumulate higher PIF levels due to impaired destabilization of these factors (Fig. 1D). Hence, in response to BRZ+GA application, higher levels of accumulation of the PIF4 factor lead to an elongation response similar to that observed for the constitutive BR response bes1-D mutant (Yin et al. 2002).

In line with such a BR-related phenotype, a significant overlap is observed between the genes differentially expressed in PIF4-OE lines grown in red light (De Lucas et al. 2008) and in dark-grown pif $q$ seedlings (for dark data set, see Leivar et al. 2009; for pifq data set, see Zhang et al. 2013) and those induced by BL treatment (Goda et al. 2004, 2008; Nemhauser et al. 2004, 2006; Vert et al. 2005) or down-regulated in bri1-116 seedlings (Sun et al. 2010). As shown in Figure 1E, $24.9 \%$ of the up-regulated genes in PIF4-OE seedlings and $21.1 \%$ of the genes down-regulated in dark-grown pif $q$ seedlings are positively regulated by BL (4.8\% expected randomly). Notably, genes involved in cell wall modification (pectinesterases, XTHs, and expansins) and auxin response (SAURs and Aux/IAA) are highly enriched among the coregulated genes, in agreement with recent studies establishing that PIF4 and BZR1 cooperatively regulate these gene targets (Oh et al. 2012).

\section{PIF4 interacts with the BR signaling kinase BIN2}

The GSK3-like kinase BIN2 negatively regulates BR signaling by inhibiting BES1/BZR1 transcriptional activity. BIN2-mediated phosphorylation of these factors inhibits DNA binding and promotes BES1/BZR1 interaction with cytosolic 14-3-3 proteins in addition to induce their degradation by the proteasome (Zhao et al. 2002; Vert and Chory 2006; Gampala et al. 2007). BR signaling 

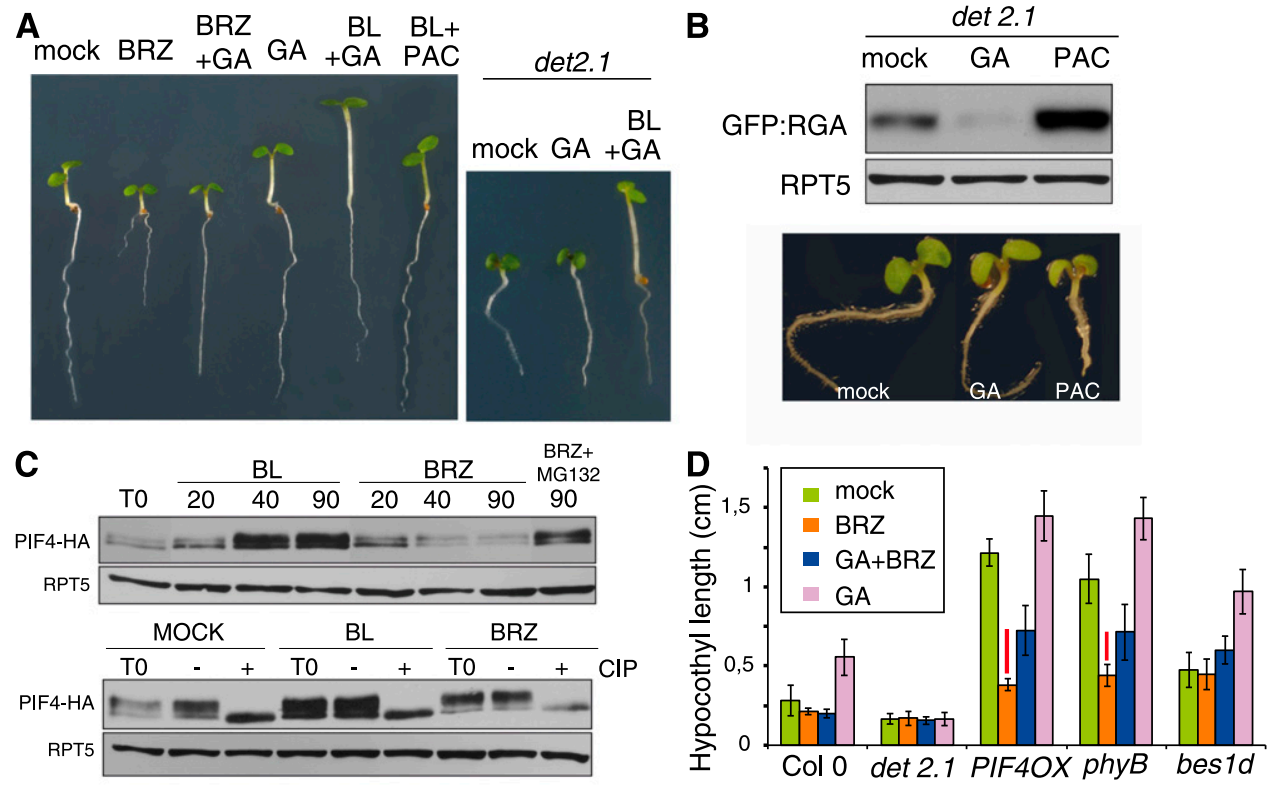

B
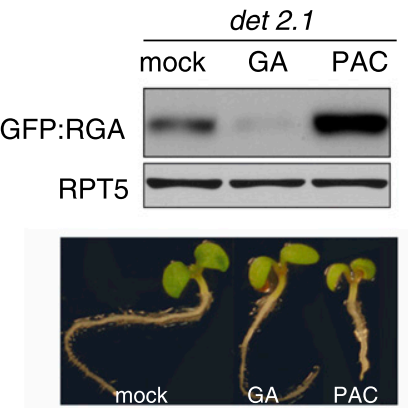

E
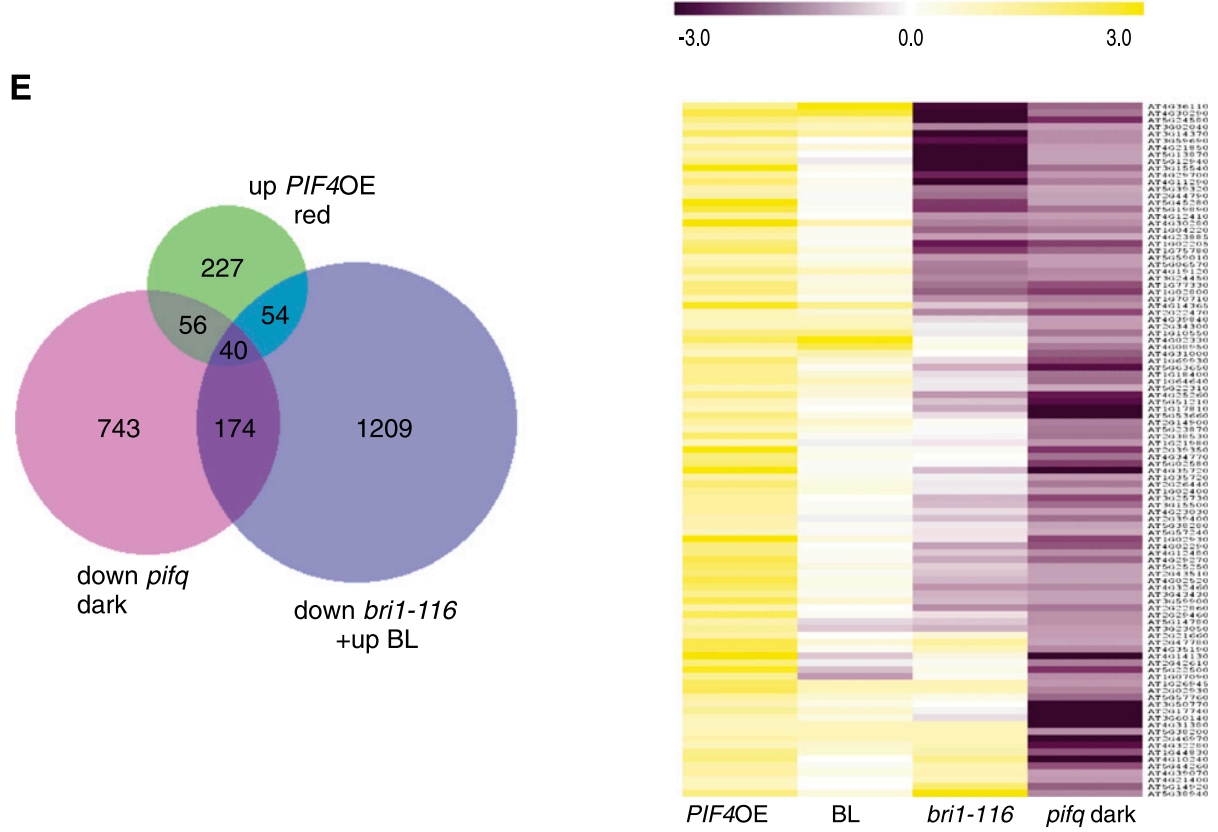

Figure 1. BR signaling overcomes the growth-repressing effects of DELLAs. (A) Hypocotyl lengths of 5-d-old wild-type (Col-0) and det2-1 seedlings grown on MS medium supplemented with mock solution (mock), $0.5 \mu \mathrm{M} \mathrm{BRZ,} 0.5 \mu \mathrm{M}$ BRZ $+25 \mu \mathrm{M}$ GA 3 (BRZ+GA), $25 \mu \mathrm{M} \mathrm{GA}_{3}(\mathrm{GA}), 1 \mu \mathrm{M}$ epi-BL $+25 \mu \mathrm{M} \mathrm{GA}_{3}(\mathrm{BL}+\mathrm{GA})$, and $1 \mu \mathrm{M}$ epi-BL $+0.5 \mu \mathrm{M}$ PAC (BL+PAC). BRZ-treated wild-type seedlings and the BR-deficient det2-1 mutants are insensitive to GA application. Combined application of GA+BL leads to exaggerated hypocotyl growth of wild-type and det2-1 seedlings. (B) GA-induced destabilization of the GFP-RGA fusion in 5-d-old det2-1 pRGA::GFP-RGA seedlings. Application of the inhibitor PAC leads to increased levels of accumulation of this DELLA and additional growth inhibition of these plants. GA induces degradation of the RGA repressor but does not promote growth of the BR-deficient seedlings. (C) BL-induced stabilization of the PIF4-HA protein. An anti-HA antibody was used for Western immunoblot detection of the PIF4-HA protein. (Top panel)Five-day-old dark-grown 35S: :PIF4-HA seedlings were kept on MS medium (mock) or treated with $1 \mu \mathrm{M}$ BL (BL), $0.5 \mu \mathrm{M}$ BRZ (BRZ), or $0.5 \mu \mathrm{M} \mathrm{BRZ} \mathrm{+} 10 \mu \mathrm{M}$ MG132 (BRZ+MG132) for the indicated times. A faster-migrating band of the protein is found to be enriched in the BL-treated plant. (Bottom panel) Five-day-old dark-grown seedlings were treated overnight with mock solution (mock), $1 \mu \mathrm{M} \mathrm{BL}(\mathrm{BL})$, or $0.5 \mu \mathrm{M} \mathrm{BRZ}$ (BRZ), and total protein extracts were incubated for $1 \mathrm{~h}$ with $(+)$ or without $(-)$ calf intestinal phosphatase (CIP). A fraction of the extract was sampled before incubation (TO). Slower-migrating forms of the protein disappear after CIP treatment, indicating that they correspond to phosphorylated forms of PIF4. (D) BL response phenotype of PIF4OX and phyB plants. Seedlings were grown in the light on vertical MS plates supplemented with mock solution (mock), $0.5 \mu \mathrm{M}$ BRZ (BRZ), $0.5 \mu \mathrm{M}$ BRZ $+25 \mu \mathrm{M}$ GA 3 (BRZ+GA), or $25 \mu \mathrm{M} \mathrm{GA}_{3}(\mathrm{GA})$, and hypocotyl lengths were measured $(n=20)$ at day 5 after germination. Error bars are \pm SEM. $(E)$ PIF4-induced genes are positively regulated by BRs. Venn diagram showing the overlap among genes induced in PIF4OX transgenic plants or repressed in the pif $q$ mutant and BR-regulated genes (induced by BL treatment or repressed in the bri1-116 mutant). Clustering analysis of FC expression of the genes coregulated in PIF4OX plants grown in red light (PIF4OX) with $3 \mathrm{~h}$ of BL treatment (BL) and the bri1-116 mutant (bri1-116) and pifq seedlings grown in the dark (pifq) ([yellow] up; [purple] down). 
inactivates BIN2 kinase activity via BSU1-mediated dephosphorylation at Tyr200, enabling nuclear accumulation of the nonphosphorylated BES1/BZR1 factors and BR-activated gene expression (Kim and Wang 2010). Thus, BIN2 is a good candidate to regulate PIF4 levels, as inactivation of this kinase by $\mathrm{BL}$ may result in the fastest-migrating form of this factor seen in BL-treated seedlings (Fig. 1C). A direct interaction of the full-length BIN2 protein and PIF4 was actually observed in yeast two-hybrid assays (Fig. 2A). Deleted versions of the PIF4 protein mapped the BIN2 interaction domain to a fragment immediately C-terminal to the bHLH dimerization domain, as a PIF4 truncated version from Val312 to the Cterminal end still interacts with BIN2 (Fig. 3D). Deletion of this C-terminal end, however, does not abrogate binding, suggesting that an additional interacting domain located between residues 107 and 312 cooperates with the C-terminal region to enhance BIN2-binding affinity (Fig. 3D).

Direct interaction of these proteins was confirmed by in vitro pull-down assays in which the ${ }^{35 \mathrm{~S}}$ Met-labeled PIF4 and BES1 were found to be retained with similar affinities by agarose beads with the immobilized GST-BIN2 protein (Fig. 2B). Bimolecular fluorescence complementation (BiFC) and coimmunoprecipitation (co-IP) studies in Nicotiana benthamiana leaves further confirmed this interaction in vivo. Nuclear fluorescence of reconstituted split YFP was observed in leaves cotransfected with the BIN2-eYFC and PIF4-eYFN fusions but not in control leaves expressing the eYFC-BIN2 and eYFN or the eYFC and PIF4-eYFN proteins (Fig. 2C). The PIF4-GFP protein was also pulled down out of plant extracts coexpressing the BIN2-HA and PIF4-GFP proteins after immunoprecipitation of BIN2HA (Fig. 2D), confirming interaction of these proteins in plant cells.

\section{BIN2 phosphorylates PIF4 in vitro}

In vitro kinase assays using the purified GST-BIN2, PIF4$6 \times$ His, and MBP-BES1 proteins showed that BIN2 is able to phosphorylate both BES1 and PIF4 (Fig. 3A). Labeling of these factors is only observed when BIN2 is added to the reaction mix. Also, although in pull-down assays BIN2 bound with similar affinities the BES1 and PIF4 proteins, labeling of PIF4 was weaker than that of the BES1 protein. This would suggest that PIF4 has fewer phosphorylation sites than BES1, reported to be modified by this kinase in at least 10 independent Ser/Thr residues (Ryu et al. 2010). Incubation with the specific inhibitor bikinin (De Rybel et al. 2009) blocked PIF4 phosphorylation as well as autophosphorylation of BIN2, confirming that labeling of this protein is mediated by the BIN2 kinase (Fig. 3C). Therefore, we can conclude that PIF4 is a bona fide substrate for the BR signaling kinase BIN2.

\section{Mutation of a conserved BIN2 phosphorylation consensus leads to strong PIF4 stabilization}

GSK3-kinases phosphorylate Ser/Thr residues in the short consensus motif (S/T)-X-X-X-(S/T). Identification of the BES1/BZR1 factors as main BIN2 phosphorylation substrates revealed that this plant kinase phosphorylates Ser/Thr residues within the same motif (Ryu et al. 2010). However, in contrast to animal GSK3-kinases, it does not require a priming phosphorylation event to act on these proteins (Peng et al. 2010). In a search for residues that match this consensus motif in the PIF4 protein, we identified $>10$ putative phosphorylation sites (Supplemental Fig. S5). We reasoned that if these sites are important to modulate PIF4 stability, they should be conserved in all PIF4 orthologs. Alignment of the Arabidopsis protein with its closest homologs in other species actually
A
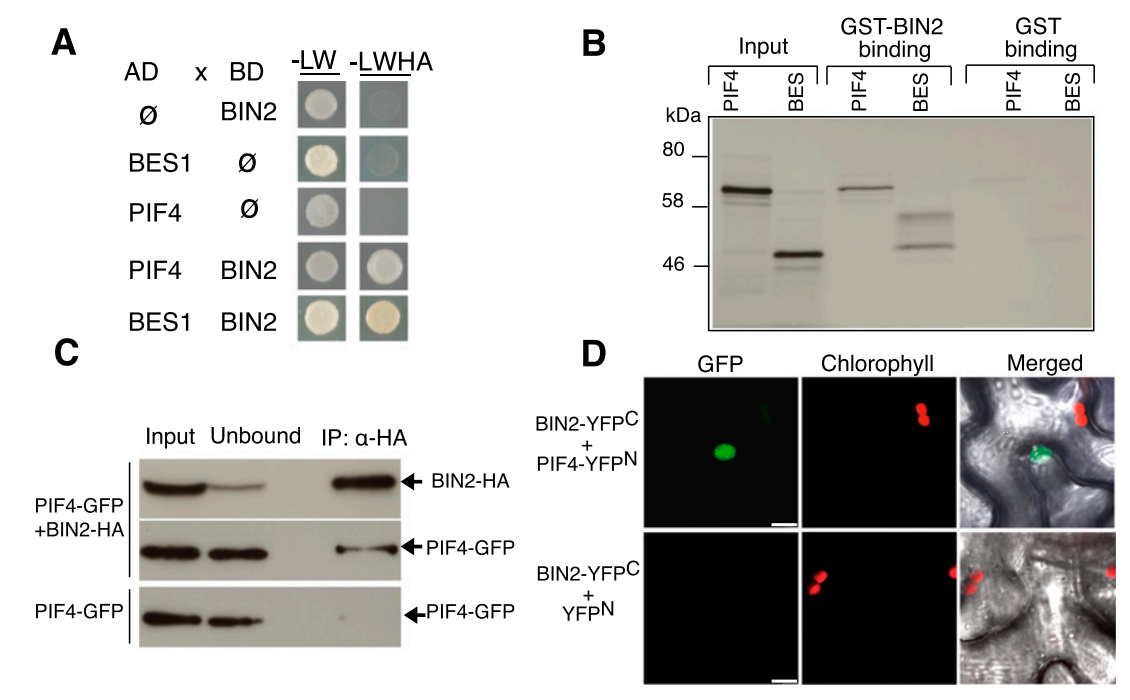
struct and the empty eYFP ${ }^{\mathrm{N}}$ vector, used as negative control. In red is shown the autofluorescence of chloroplast. $(D)$ Co-IP of the BIN2HA and PIF4-GFP proteins. Leaf extracts of $N$. benthamiana plants coexpressing these protein constructs were immunoprecipitated with an anti-HA antibody, and the immunoblots were probed with anti-GFP and anti-HA antibodies. (Bottom panel) A signal corresponding to the PIF4-GFP protein was not observed after immunoprecipitation of extracts expressing the PIF4-GFP protein alone.

Figure 2. PIF4 interacts with the BR pathway kinase BIN2 in vitro and in vivo. (A) Yeast twohybrid assay showing PIF4 and BIN2 interaction. Bait $(\mathrm{BD})$ and prey $(\mathrm{AD})$ constructs were cotransformed into yeast cells as indicated. Growth of yeast cells on SD-His Ade medium indicates interaction. The BIN2-BD and BES1$\mathrm{AD}$ interaction is shown as a positive control for binding. $(B)$ In vitro pull-down assay for BIN2/PIF4 interaction. A glutathione-Sepharose matrix with the bound GST-BIN2 protein retained with similar affinities the in vitro translated ${ }^{35}$ S-PIF4 and ${ }^{35}$ S-BES1 proteins. Only background levels of these proteins are detected after incubation with a GST matrix. (C) BiFC PIF4/BIN2 interaction assay. The BIN2-eYFP ${ }^{\mathrm{C}}$ and PIF4-eYFP ${ }^{\mathrm{N}}$ constructs were coinfiltrated in $N$. benthamiana leaves. Nuclear GFP fluorescence was observed in cells infiltrated with these two constructs but not in cells infiltrated with the BIN2-eYFC con- 
A

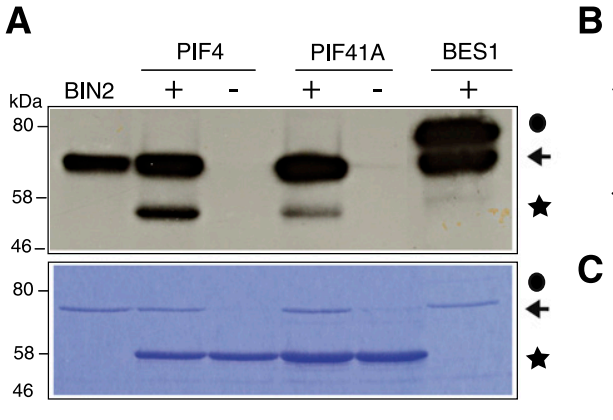

D

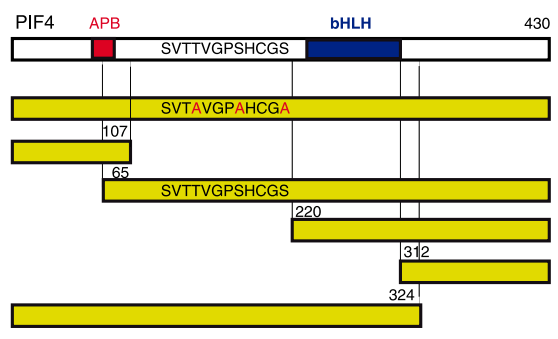

B 462 CTCGGTAACGACCGTTGGACCTAGCCATTGCGGAAGCAA

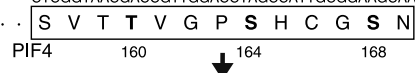
${ }^{462}$ CTCGGTAACGGCCGTTGGACCTGCCCATTGCGGAGCCAA \begin{tabular}{|cccccccccccccc}
\hline S & $V$ & T & A & $V$ & $G$ & P & A & H & C & G & A & N. \\
\hline
\end{tabular}

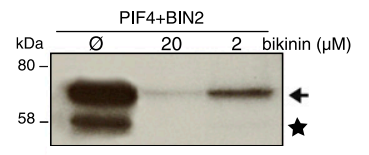

E

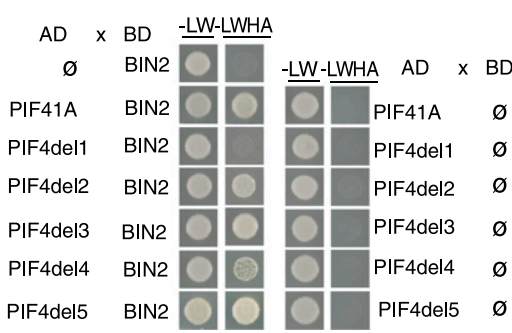

Figure 3. BIN2 phosphorylates PIF4 in in vitro kinase assays. The PIF4 and BIN2 interaction is mediated by the bHLH PIF4 domain and residues immediately adjacent to this domain. $(A)$ BIN2 phosphorylates PIF4 in vitro. PIF4-6xHis, PIF41A-6xHis, and MBP-BES1 were incubated alone or with the BIN2 kinase fused to GST and ${ }^{32} \mathrm{P}-\gamma$ ATP. The top panel shows the autoradiogram, and the bottom panel shows the Coomassie brilliant blue (CBB) staining of the gel. Mutation of Thr/Ser residues in the conserved BIN2 phosphorylation reduces labeling efficiency. The BES1 protein has multiple phosphorylation sites and is more efficiently labeled in these assays. Positions of the BIN2, BES1, and PIF4 proteins are marked by an arrow (BIN2), a circle (BES1), and a star (PIF4). (B) Representation of the conserved $\underline{T}^{160}$ VGPS ${ }^{164}{ }^{H C G} \underline{S}^{168}$ motif in which the Ser/Thr residues were replaced by Ala to generate the nonphosphorylatable PIF41A mutant protein. Mutation of these residues decreases the efficiency of labeling by the kinase BIN2 (shown in $A$ ). $(C)$ Inhibition of PIF4 phosphorylation by the BIN2 kinase inhibitor bikinin. Addition of $2 \mu \mathrm{M}$ and $20 \mu \mathrm{M}$ bikinin to the phosphorylation mix represses PIF4 phosphorylation and the autophosphorylation activity of BIN2. (D) Mapping of the BIN2-interacting domain. Schematic representation of the PIF4 domains and the deletions used in the yeast two-hybrid assay are shown. $(E)$ Deletions of the PIF4 protein were cotransformed into yeast cells with the BIN2-BD construct to map the interacting domain. A C-terminal fragment lacking the bHLH domain binds the BIN2 protein, although with lower affinity. Removal of this C-terminal region does not abrogate binding, indicating that the HLH region contributes to this interaction.

identified two consensus motifs that are conserved in all PIF4/PIF5 proteins across evolutionarily divergent species (Supplemental Fig. S5). Site-directed mutagenesis of the conserved $\underline{T}^{160} \mathrm{VGP} \underline{S}^{164} \mathrm{HCG} \underline{S}^{168}$ motif to replace the three Ser/Thr residues by Ala, hence generating a nonphosphorylatable PIF41A mutant protein (Fig. 3B), led to a strong stabilization of this factor (Fig. 4B,D) and reduced the efficiency of in vitro labeling by BIN2 (Fig. 3A), although it did not fully abolish phosphorylation. Residual labeling by this kinase is likely to be due to phosphorylation of the second conserved motif or secondary modification of the nonconserved sites found in the protein. However, further mutation of $\mathrm{Ser}^{202}$ to Ala to disrupt this second conserved motif did not lead to protein stabilization, indicating that this motif is less relevant for PIF4 function.

Consistent with these results, lines overexpressing the PIF41A-GFP protein fusion were severely elongated and showed extremely early flowering (Fig. 4A), as anticipated for a strong stabilization of this factor. Confocal GFP fluorescence and Western blot studies confirmed higher levels of accumulation of this mutant protein fusion in the light (Fig. 4B,D), as compared with 35S: PIF4-GFP lines showing equivalent transcript levels for the wildtype protein fusion (Fig. 4C). In dark-grown seedlings, differences in protein levels were smaller due to additional stabilization of the wild-type protein (Fig. 4D). However, subcellular localization of the PIF41A-GFP protein was not altered with respect to PIF4-GFP. Both the PIF4-GFP and PIF41A-GFP fusions show a diffuse nuclear expression and form similar nuclear speckles upon red light irradiation
(Bauer et al. 2004; Leivar et al. 2008), with a pulse of far-red light reversing speckle formation in both proteins (Supplemental Fig. 6). BL treatment, on the other hand, did not alter their subcellular localization (Supplemental Fig. 6).

35S: :PIF41A-GFP lines display up-regulated expression of the PIL1 transcript and respond to BL by enhanced levels of activation of this gene (Fig. 4E). When treated with BRZ, PIL1 gene expression was also found to be less suppressed in these plants than in 35S: :PIF4-GFP lines (Fig. 4E), suggestive of a stabilization of the PIF41A protein irrespective of BIN2 kinase activation.

\section{PIF41A expression partially suppresses the dwarf phenotype of bin2-1 mutants}

To further assess constitutive activation of this mutant protein, we analyzed whether PIF41A overexpression suppresses the severe dwarf phenotype of bin2-1 mutants, impaired in BR signaling due to constitutive BIN2 activation (Peng et al. 2008). As shown in Supplemental Figures S9 and S10, PIF41A expression rescued the semidwarf phenotype of heterozygous bin2-1 $1^{+/-}$mutants but did not suppress the more severe phenotype of homozygous plants. PIF41A bin2-1 seedlings are slightly taller than the bin2-1 mutant (Supplemental Fig. S10) but still display the characteristic curled leaf and severe dwarf phenotype of these mutants. Such dosage complementation effects indicate that, in addition to PIF4, other BIN2-regulated factors are required for hypocotyl growth, in agreement with the recent finding that BZR1 binds G-box elements identical to those bound by PIF4 in the promoters of cell 
A

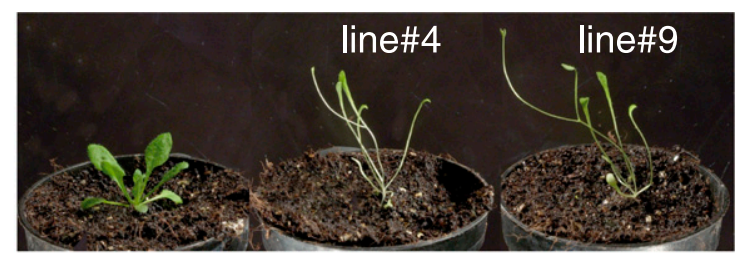

PIF4-GFP

B

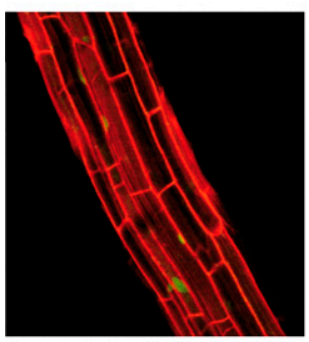

PIF4-GFP
PIF41A-GFP

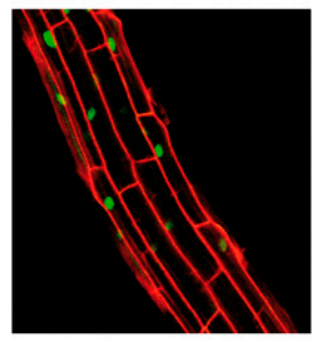

PIF41A-GFP

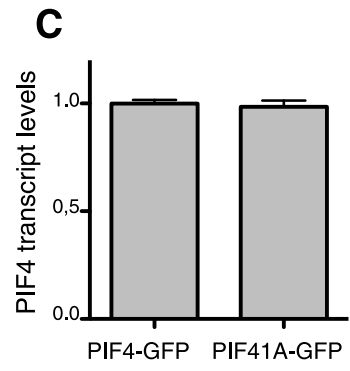

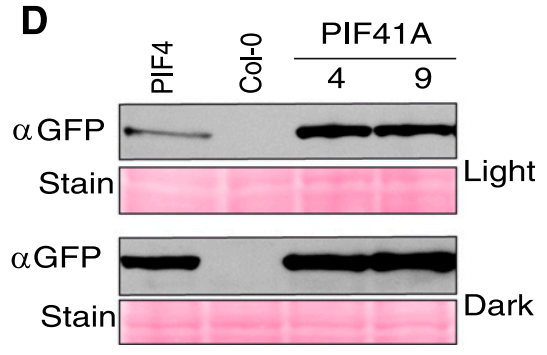

E

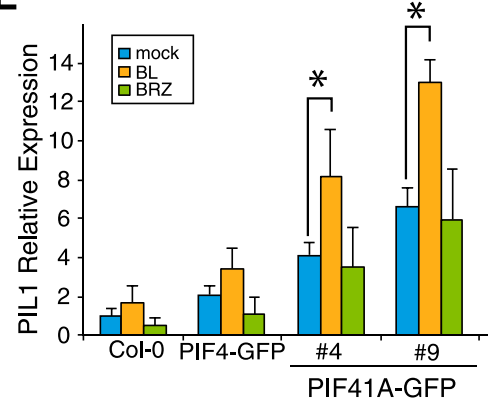

Figure 4. PIF41A overexpression causes a severely elongated phenotype and activated PIL1 gene expression in the presence of BRZ. $(A)$ Overexpression of the PIF41A mutant protein causes severe elongation of the petioles and stem and an extremely early flowering phenotype. Plants were grown for 3 wk. $(B)$ Detection of the PIF1A-GFP protein in roots of 5-d-old plants. Nuclear fluorescence in PIF41A-GFP plants is stronger than in PIF4-GFP seedlings, indicating a higher stability of the PIF41A protein. $(C)$ Quantification of PIF4 transcript levels in the PIF4-GFP and PIF41A-GFP overexpression lines. Plants were grown in continuous light for $5 \mathrm{~d}$. Transcript levels were equivalent in both lines. $(D)$ Western blot detection of the PIF4-GFP and PIF41A-GFP proteins. Plants were grown for $5 \mathrm{~d}$ under light or dark conditions. Higher levels of PIF41A are detected in light-grown seedlings, implicating that phosphorylation by BIN2 mediates destabilization of this factor in the light. $(E)$ Quantitative real-time PCR analysis of PIL1 transcript levels in Col-0 and in lines overexpressing the PIF4 and PIF41A proteins. Plants were grown in continuous light for $5 \mathrm{~d}$. An enhanced activation of PIL1 in response to BL was observed in PIF41A seedlings. $\left(^{\star}\right) P<$ 0.05 by Student's $t$-test. Error bars are \pm SD. elongation genes (Oh et al. 2012). PIFs are actually required for BZR1-induced cell elongation, with loss-of-function mutations of the PIF1, PIF3, PIF4, and PIF5 genes in the pif $q$ mutant suppressing the elongated phenotype of the constitutive bzr1-1D mutant (Oh et al. 2012). Hence, concurrent activity of the BZR1 and PIF4 factors is essential for cell elongation. The observation that bzr1-1D suppresses the dwarf phenotype of bri1-116 seedlings in the dark but not in the light and that light-grown PIF4OX bzr1-1D bri1-116 seedlings are shorter than PIF4OX lines indeed supports this regulatory model and demonstrates that phosphorylation by the BR signaling kinase BIN2 plays an important role in modulating PIF4 stability and in BR promotion of cell elongation in the light.

\section{BIN2 phosphorylation plays a main role in destabilizing the PIF4 protein at dawn}

Growth of young seedlings follows a rhythmic elongation pattern such that, in short days, rapid hypocotyl growth occurs at dawn and alternates with an interval of reduced elongation during daytime and early night (Nozue and Maloof 2006). Thus, we wanted to test whether PIF4 phosphorylation plays a role in defining this window of active cell elongation. To this aim, we generated transgenic pif4pif5 lines that expressed the wild-type PIF4-HA and mutant PIF41A-HA proteins under the control of its native promoter. We selected lines in which the levels of expression of these transgenes were similar to Col-0 and verified that transcript accumulation in these plants (Fig. 5D) followed the diurnal expression pattern reported for the endogenous PIF4 gene (Nozue et al. 2007; Yamashino et al. 2013). Lines generated for the wild-type PIF4-HA protein actually had hypocotyl lengths similar to those of Col-0 plants and showed a wild-type response to PAC (Supplemental Fig. S12) and BRZ treatments (Fig. 7A,B, below). When grown at $28^{\circ} \mathrm{C}$, these plants also showed an elongation growth similar to that observed for Col-0 seedlings (Supplemental Fig. S7). PIF41A-HA lines, in contrast, had taller hypocotyls and showed the characteristic elongated petioles and narrower leaves of plants grown in the shade (Figs. 5A, 7A [below]; Supplemental Fig. S7), indicative of an increased stability of this mutant protein. Indeed, PIF41A-HA was found to accumulate to higher levels than wild-type PIF4-HA, with differences in protein levels being greatest at Zeitgeber time 22 (ZT22) and ZT2, coinciding with the interval of maximal hypocotyl growth (Fig. 5B,C). PIF41A levels, in addition, did not change in response to $\mathrm{BL}$ or $\mathrm{BRZ}$ treatments, demonstrating that this mutation results in a stabilization of the protein irrespective of BR signaling (Supplemental Fig. S8).

Notably, although PIFs are accepted to be destabilized by PHYB in the light, both PIF4 and PIF41A proteins were detected to significant levels during daytime and rapidly 
A

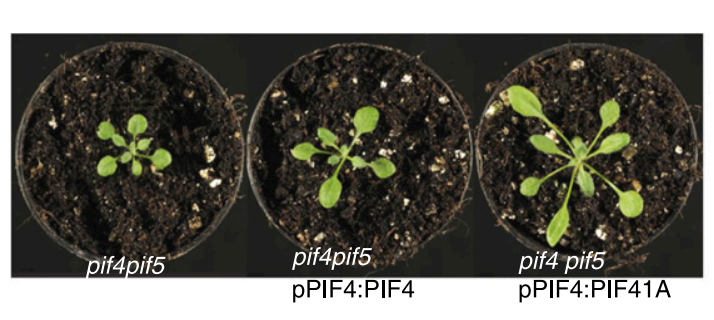

B

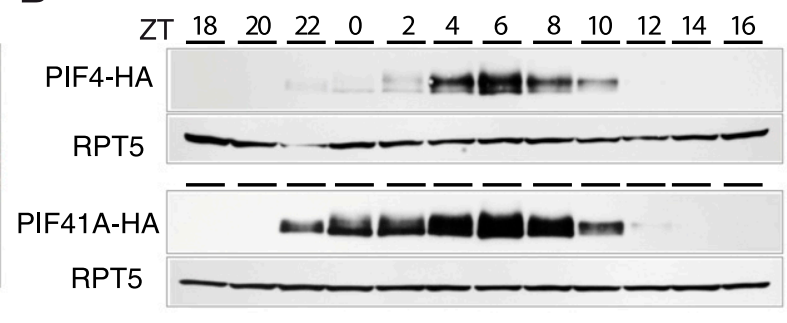

C

$1 \mathrm{~A}$

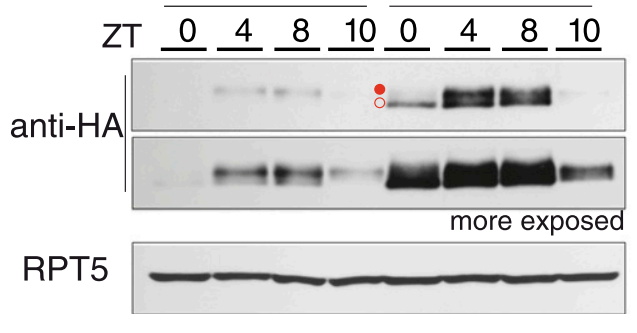

D

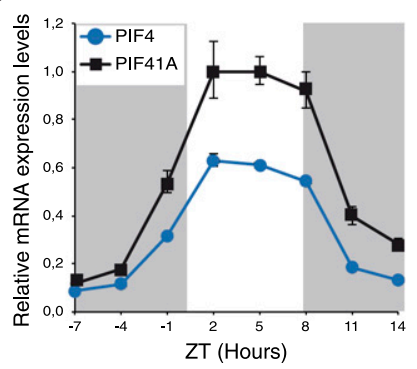

$\mathbf{E}$

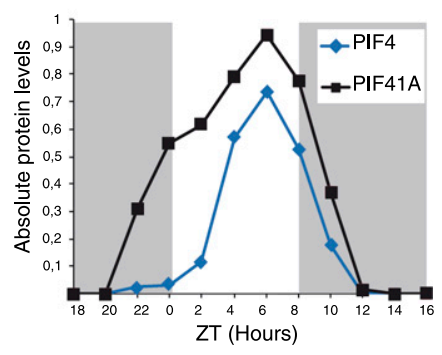

Figure 5. BIN2 modulates PIF4 stability at dawn. (A) Phenotype of pif4pif5 and transgenic pif4pif5 lines expressing the wild-type PIF4HA and mutant PIF41A-HA proteins under the control of its native promoter. pPIF4: PIF41A-HA pif4pif5 lines show elongated petioles and narrow leaves, characteristic of plants grown in the shade. Plants were grown for 3 wk. $(B)$ Levels of accumulation of the PIF4-HA and PIF41A-HA proteins. Plants were grown in short-day conditions for $6 \mathrm{~d}$, and samples were collected every $2 \mathrm{~h}$ during a cycle of $24 \mathrm{~h}$. Levels of the proteins were analyzed by Western blot using an antibody against HA. PIF4-HA and PIF41A-HA accumulated at the end of the night and during daytime and dropped after dusk, with PIF41A found to be more stable than the wild-type PIF4-HA protein. An antibody against RPT5 was used as a loading control. $(C)$ To compare stability of these proteins, samples corresponding to selected points were run in the same gel. (D) Analysis of PIF4 transcript levels in these plants. Plants were grown in short-day conditions for $5 \mathrm{~d}$. Samples were collected every $3 \mathrm{~h}$ during a cycle of $24 \mathrm{~h}$. Error bars represent \pm SEM of two independent biological replicates. (E) Quantification of the PIF4-HA and PIF41A-HA proteins in the Western blots shown in B. Intensities of the detected bands were quantified using the ImageJ program. PIF41A accumulates to higher levels than PIF4, particularly during late night (ZT22) and early morning (ZT0-ZT2).

dropped after dusk, when PIF4 transcription is repressed (Fig. 5B,D; Nusinow et al. 2011). A related daytime pattern of protein accumulation was also recently reported for a PIF4-citrine-HA construct (Yamashino et al. 2013), although the taller phenotype of these plants did not exclude an additional stabilization of the protein due to the C-terminal citrine tag. Our HA-tagged lines display a hypocotyl length similar to that of Col-0, and relatively high levels of the protein were detected during daytime. Furthermore, in lines expressing the same PIF4-HA fusion under the control of the $35 \mathrm{~S}$ promoter (Supplemental Figure S11), a preferential accumulation of the protein was seen at night, as previously reported (Nozue et al. 2007). This indicates that although stability of PIF4 is higher in darkness, active transcription of this gene may lead to substantial levels of accumulation of this factor during daytime, depending on light irradiance. However, during this phase of the day, PIF4 is repressed by the DELLAs, which were reported to accumulate in the light (Achard et al. 2007). It is also noteworthy that increased PIF41A stability during late night correlates with preferential accumulation as a faster-migrating form of the protein (Figs. 5B, 1C), although this mutation does not preclude formation of slower isoforms, suggesting that PIF4 is phosphorylated by other kinases in addition to BIN2. Indeed, upon light exposure, slower-migrating forms are observed for both the wild-type and PIF41A proteins (Fig. 5B), suggesting that PIF4 is subjected to phosphorylation by a PHYB-regulated kinase, as reported for PIF3 (Ni et al. 2013).

To estimate the stabilization of this mutant protein, we treated PIF4 and PIF41A seedlings with cycloheximide (CHX) and with CHX and BL (Fig. 6A). In these studies, PIF4 is destabilized by $30 \mathrm{~min}$ of CHX application, while PIF41A is still detectable after 90 min of CHX treatment. Also, upon BL application, PIF4 half-life was similar to that of the PIF41A protein, whereas this hormone did not have any effect on PIF41A protein levels (Fig. 6A). Consistent with these results, when PIF4 and PIF41A lines were crossed to the bin2.1 mutant, we observed that constitutive activation of BIN2 in these seedlings leads to a strong reduction in PIF4 protein levels but has only a minor effect on PIF41A protein stability (Fig. 6B).

We also assessed whether this mutation affects rhythmic plant growth by continuously measuring the growth of Col-0, PIF4-HA, PIF41A-HA, and PIF4OX seedlings during $2 \mathrm{~d}$ (Fig. 6C). Under short days, growth of Col-0 and PIF4-HA was maximal at dawn, while PIF4OX seedlings showed an increased growth rate during early night, as previously described (Nozue et al. 2007). Remarkably, PIF41A seedlings elongated also during daytime (Fig. 6C), indicating that BL-dependent phosphorylation of the PIF4 factor plays an important role in modulating rhythmic hypocotyl growth. 
PIF41A bes1-D seedlings are insensitive to BRZ in the light

The gain-of-function bes1-D and bzr1-1D mutations cause constitutive BES1 and BZR1 activation due to identical mutations in the PEST domain, which enhance PP2Abinding affinity and lead to increased dephosphorylation of these factors (Tang et al. 2011). These mutants show a BRZinsensitive response in the dark but opposite phenotypes in the light, although the BES1 and BZR1 proteins share $88 \%$ overall identity. While the bzr1-1D mutant displays a weak dwarf phenotype and a hypersensitive response to BRZ in the light, light-grown bes1-D seedlings are largely insensitive to this inhibitor. This phenotype is at present not well understood, as light does not seem to affect levels of these proteins (Luo et al. 2010). Since BES1 and BZR1 promote cell elongation by formation of a coactivator complex with PIF4 (Oh et al. 2012), it is possible that the hypersensitive response to BRZ of bzr1-1D seedlings results from an increased phosphorylation and destabilization of the PIF4 factor in the light. However, the BRZ-insensitive response of light-grown bes1-D mutants does not fit well with this model, unless these plants still show a reduction in hypocotyl growth on BRZ. As shown in Figure 7, A and $\mathrm{B}$, bes1-D seedlings are in fact shorter on BRZ than in mock medium and display reduced levels of expression of the PIL1 and PRE5 target genes (Fig. 7C). Also, they are only slightly taller than Col-0, although BES1 is constitutively activated in this mutant. PIF41A lines, in contrast, show a much elongated phenotype but still respond to BRZ by a reduction in hypocotyl growth, indicating that the nonphosphorylatable PIF41A protein requires BES1/BZR1 function to promote cell elongation. Therefore, we introduced the bes1- $D$ gain-of-function mutation into the pPIF4: PIF41A lines to test whether combined expression of these two mutant proteins confers a BRZ-insensitive response in the light. Light-grown PIF41A bes1- $D$ seedlings actually show elongated hypocotyls on BRZ similar to those in mock treatments (Fig. 7B), demonstrating that the BIN2-insensitive forms of PIF4 and BES1 are required for hypocotyl growth in the light. Gene expression quantitative PCR (qPCR) studies confirmed that expression of both PIF41A and bes1-D proteins leads to constitutive upregulated levels of expression of the PIL1, XTR7, and PRE5 target genes on BRZ (Fig. 7C). Taken together, these results demonstrate that $\mathrm{BR}$ signaling plays a prevalent role in hypocotyl elongation by suppressing BIN2 kinase activity, with inactivation of this kinase allowing stabilization of the PIF4 factor and BES1/BZR1 nuclear accumulation, with a concerted activation of these two families of regulators being essential for growth.

\section{Discussion}

PIFs play a pivotal role in cell elongation by directly activating the expression of genes with a role in cell wall loosening and auxin-related signaling (Leivar and Quail 2011). Members of this gene family play a redundant role in seedling etiolation and loss of function of the PIF1, PIF3, PIF4, and PIF5 genes in the pifq mutant, leading to a dwarf de-etiolated phenotype in the dark (Leivar et al. 2009). PIF4 was recently shown to interact with BES1/BZR1 and act in an interdependent way with these factors to activate expression of multiple BR-regulated genes (Oh et al. 2012). In this study, we disclose a role of BIN2 in destabilization of the PIF4 factor and show that this modification plays a key role in timing seedling elongation to the end of the night. We show that active BR signaling is required for growth promotion in response to DELLA destabilization and that BR application promotes accumulation of a faster-migrating form of the PIF4 protein, suggestive of a role of BRs in modulating phosphorylation of this factor. PIF4 binds the negative BR signaling kinase BIN2 in yeast cells, and interaction of these proteins was confirmed in vivo by BiFC and co-IP assays. BIN2 phosphorylates the PIF4 protein in vitro, and we show that this modification plays an important role in modulating stability of this factor.

\section{BR signaling stabilizes the PIF4 factor}

Expression of the PIF41A mutant protein lacking a conserved BIN2 phosphorylation consensus leads to an elongated phenotype and reduced sensitivity to the BR biosynthetic inhibitor BRZ. However, this mutation suppresses only in part the dwarf phenotype of the BRinsensitive bin2-1 mutant, indicating that other BIN2regulated factors also play an essential role in hypocotyl growth. BIN2 phosphorylates the HLH BES1/BZR1 factors at multiple sites, the modified BES1/BZR1 proteins being unable to bind to DNA and be retained in the cytosol via interaction with the 14-3-3 phosphopeptidebinding proteins, where they are degraded by the proteasome (Zhao et al. 2002; Vert and Chory 2006; Gampala et al. 2007). BZR1 was shown to interact with PIF4 and bind as a complex with this factor to the promoters of several genes (Oh et al. 2012). Activation of these gene targets needs PIF function, as the quadruple pifq mutant suppresses the BRZ-insensitive phenotype of bzr1-1D mutants and leads to short hypocotyls, as in pif $q$ plants. Constitutive bzr1-1D mutants are insensitive to BRZ in darkness but show a hypersensitive response to this inhibitor in the light, in contrast to bes1-D mutants, reported to display also a BRZ-insensitive response in the light. Here we show that light-grown bes1-D seedlings still respond to BRZ by an inhibition of hypocotyl elongation and reduced PIL1 and PRE5 gene expression, suggesting that BIN2 phosphorylation and destabilization of PIF4 mediates this additional response to BRZ. In line with this model, expression of the PIF41A mutant protein in bes1-D plants resulted in tall plants that are fully insensitive to BRZ and show elevated levels of expression of the PIL1, XTR7, and PRE5 genes in the presence of this inhibitor. These results demonstrate that BR signaling not only activates BES1 and BZR1 but also promotes stabilization of the PIF4 factor, a concerted action of these two families of regulators being required for elongation growth.

\section{PIF4 and BES1/BZR1 accumulation is required for cell elongation}

Consistent with a role of BIN2 in regulating PIF4 stability, the bzr1-1D mutation was shown to suppress the 
dwarf phenotype of bri1-116 seedlings in the dark but not in the light. Likewise, overexpression of the wild-type PIF4 protein increases the hypocotyl length of bzr1-1D bri1-116 plants (Oh et al. 2012), but these seedlings are still much shorter than the PIF4OX lines. Enhanced PP2A activity in PP2A B subunit overexpressers, on the other hand, leads to a reduced sensitivity to BRZ similar to that of the bzr1-1D mutation but is unable to suppress the weak bri1-5 allele even though these plants accumulate the dephosphorylated BZR1 protein (Tang et al. 2011).

The BZR1-BIN2 interaction is mediated by a small docking motif (DM) located near the C-terminal end of the BZR1 protein (Peng et al. 2010). DM deletion completely eliminates BZR1 phosphorylation and leads to increased levels of accumulation of the BZR1( $\triangle \mathrm{DM})$-GFP protein. However, as we observed for the PIF41A mutation, BZR1
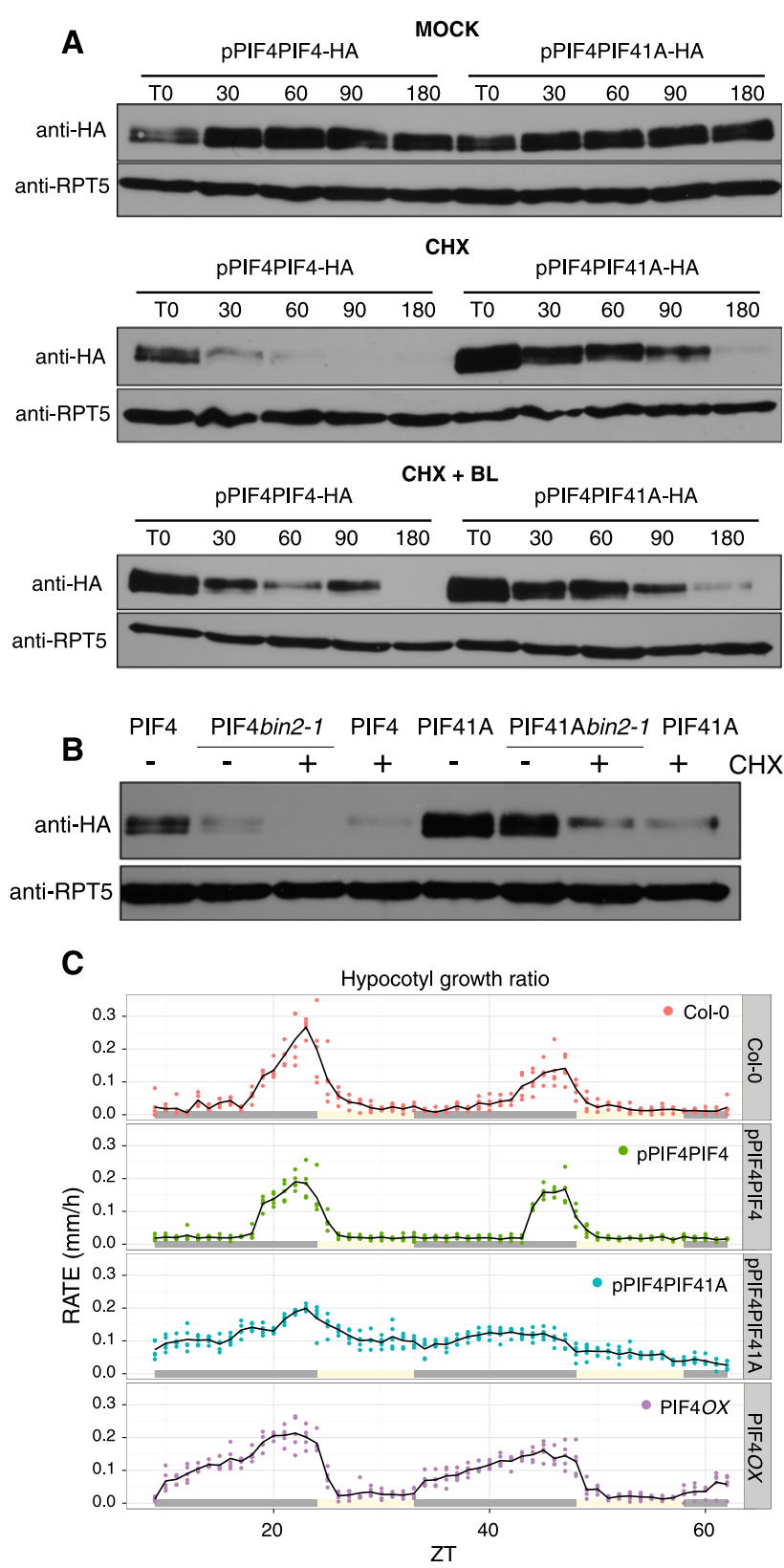

$(\Delta \mathrm{DM})$ suppresses only the weaker phenotype of bin2-1 heterozygote mutants (Peng et al. 2010). Thus, these findings support a role of BIN2 in PIF4 and BES1/BZR1 phosphorylation, nuclear accumulation of the nonphosphorylated forms of both proteins being required for growth.

\section{The concerted action of PIFs and BES1/BZR1} modulates rhythmic hypocotyl growth

Although it is widely accepted that PIFs accumulate only in darkness, we show that the PIF4 protein follows a pattern of accumulation similar to that of its transcript. We generated pif4pif5 lines expressing the PIF4-HA or PIF41A-HA proteins under the control of its native promoter and selected lines with wild-type levels of these transgenes. pPIF4: :PIF4-HA lines had a hypocotyl length and expressed the PIL1 and PRE5 genes to levels similar to those of the Col-0 plants (Fig. 7; Supplemental Figs. S7, S12). pPIF4: PIF41A-HA lines, in contrast, were much taller and showed increased levels of expression of these genes (Fig. 7). pPIF4: PIF41A plants are actually taller than the bes1-D mutant and, in the presence of BRZ,

Figure 6. Mutation of the conserved BIN2 phosphorylation motif increases the half-life of the PIF41A protein and leads to continuous growth. (A) Analysis of the half-life of the PIF4 and PIF41A proteins. Transgenic pPIF4: :PIF4-HA pif4pif5 and pPIF4: :PIF41AHA pif4pif5 seedlings were grown in SD for $5 \mathrm{~d}$ and treated for $12 \mathrm{~h}$ with $0.5 \mu \mathrm{M}$ BRZ before starting the assay. Three hours before lights on, seedlings were changed to $0.5 \mu \mathrm{M}$ BRZ (mock) or $1 \mu \mathrm{M}$ $\mathrm{BL}$ (BL) medium, and $50 \mu \mathrm{M}$ CHX was added $1 \mathrm{~h}$ later. Seedlings were kept in darkness and harvested at different times after $\mathrm{CHX}$ application, as indicated. Western blot analyses of these samples showed that the wild-type PIF4 protein is destabilized after 30 min of CHX treatment, in contrast to PIF41A, which is still detected by $90 \mathrm{~min}$ of $\mathrm{CHX}$ application. BL treatment increased PIF4 stability but did not have any effect on the PIF1A protein. Note that in BL-treated seedlings, the half-life of PIF4 becomes similar to that of PIF41A. (B) Stability of the PIF4 and PIF41A protein in the bin2. 1 mutant background. pPIF4: PIF4-HA pif4pif5 and pPIF4: :PIF41A-HA pif4pif5 lines were crossed with the bin2.1 mutant, and the accumulation levels and half-lives of these proteins were analyzed in 5-d-old plants as before. Before lights on, seedlings were transferred to MS medium with $(+)$ or without $(-)$ $50 \mu \mathrm{M}$ CHX and incubated in darkness for $30 \mathrm{~min}$. Western blot analysis showed that the wild-type PIF4 protein is destabilized in the bin2.1 mutant background but that constitutive activation of the BIN2 kinase does not affect PIF41A levels. $(C)$ Diurnal growth patterns of Col-0, pPIF4::PIF4-HA pif4pif5, pPIF4:PIF41A-HA pif4pif5, and PIF4OX plants. Seedlings were grown in SD, and images were captured $3 \mathrm{~d}$ after germination at 1 -h intervals. Five seedlings were measured for each genotype, and the growth rate was plotted as a function of time. Growth of pPIF4: PIF4-HA pif4pif5 lines was similar to the wild type, with maximal hypocotyl elongation observed at dawn (Col-0). 35S: PIF4-HA lines (PIF4OX) elongated during the whole-night period, as previously described (Nozue et al. 2007). pPIF4: PIFA1A-HA pif4pif5 lines displayed continuous growth, with the hypocotyls of these plants elongating also during daytime. Measurements were done twice with similar results. Light and dark hours are indicated by white and gray bars, respectively. 
A

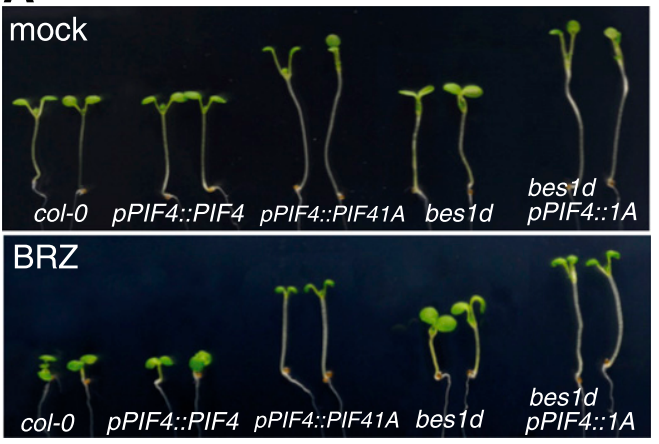

B

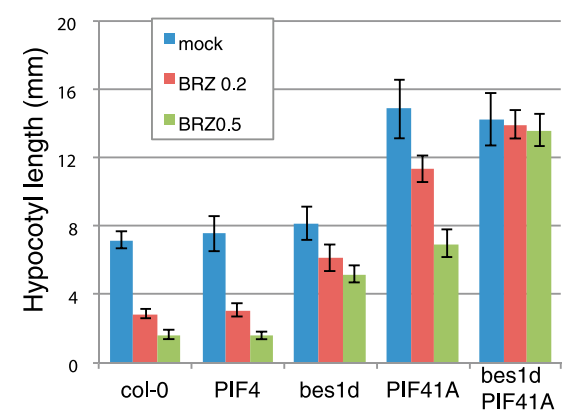

C

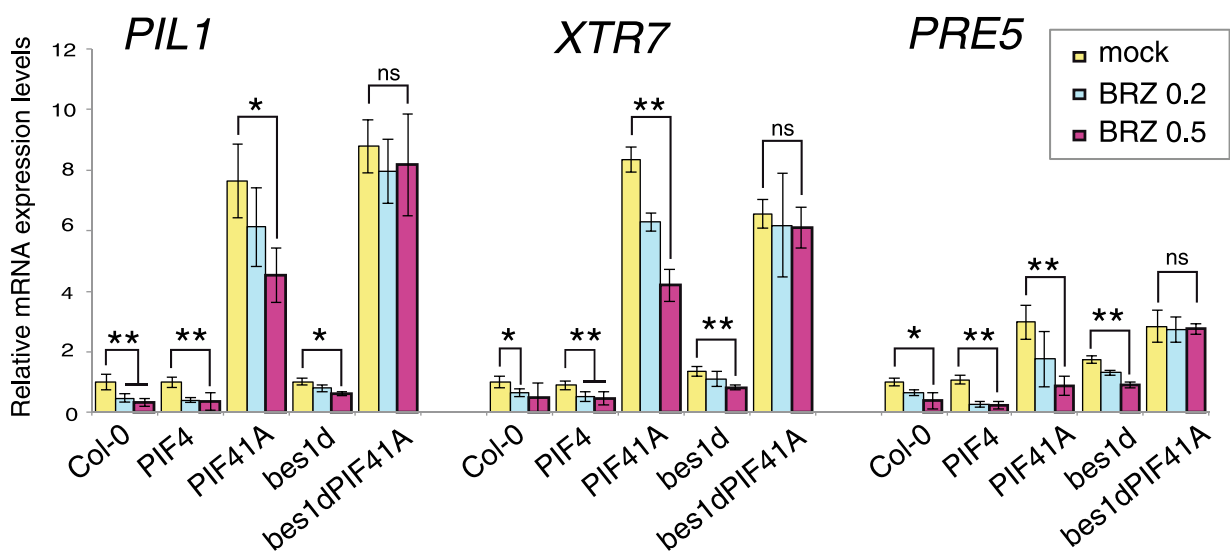

Figure 7. PIF41A bes1- $d$ seedlings are insensitive to BRZ in the light. (A) Seedlings were grown for $5 \mathrm{~d}$ in continuous red light in a medium containing mock solution (top panel) or $2 \mu \mathrm{M}$ BRZ (bottom panel). bes1-D pPIF4: PIF41A seedlings are insensitive to BRZ, while bes1-D and pPIF4:PIF4-HA plants still show a reduction in hypocotyl growth in the presence of this inhibitor. $(B)$ Hypocotyl lengths of seedlings grown on mock or BRZ medium. Seedlings were grown in vertical plates for $5 \mathrm{~d}$ in continuous red light on MS medium supplemented with mock solution or $0.2 \mu \mathrm{M}$ and $0.5 \mu \mathrm{M}$ BRZ. Error bars indicate the SD $(n=20$ plants). (C) Quantitative real-time PCR analysis of PIL1, XTR7, and PRE5 transcript levels in Col-0, bes1-D, pPIF4: PIF4-HA, and pPIF4: PIF41A-HA lines and double bes1-D pPIF4: : PIF41A-HA (bes1-D PIF41A) plants. Seedlings were grown for $5 \mathrm{~d}$ in continuous red light as before. Constitutively elevated levels of expression of the PIL1, XTR7, and PRE5 transcripts are observed in the double bes1-D PIF41A lines, demonstrating a fully BRZ-resistant response of these plants. Gene expression levels were normalized to that of $P P 2 A$ and are shown relative to the expression levels of Col-0. $\left(^{\star \star}\right) P<0.01 ;\left(^{\star}\right) P<0.05$ by Student's $t$-test. (ns) Not significant. Error bars are the \pm SEM of two biological replicates.

show increased levels of expression of the PIL1 and PRE5 transcripts, suggesting that PIF4 destabilization plays an important role in BRZ repression of hypocotyl growth. Time-course studies to analyze diurnal levels of accumulation of the PIF4 and PIF41A proteins showed that the wild-type PIF4 protein starts to accumulate at the end of the night, peaks during the day, and is reduced after transition to dark. PIF41A protein levels were higher than those of the wild-type protein, particularly during late night (ZT22) and immediately after lights on (ZT0-ZT2) (Fig. 5B). Strikingly, PIF41A plants displayed arrhythmic growth and, in contrast to PIF4 seedlings, elongated during daytime (Fig. 6C). This response is conferred by a mutation that makes PIF4 insensitive to BIN2-mediated destabilization, suggesting that diurnal oscillations in BR levels or signaling play a relevant role in setting the diurnal phase of hypocotyl growth. BR synthesis and response genes were shown to oscillate in short days, with a peak of expression that coincides with dawn (Michael et al. 2008; Yamashino et al. 2013). This time-of-day-specific expression plays a relevant role in setting the phase of maximal hypocotyl growth, as complementation of the bri1-116 mutant with the AtML1::BRI1 construct led to taller hypocotyls than the wild-type due to misexpression of the BRI1 gene during early night (Michael et al. 2008). This indicates that BR signaling plays an important role in hypocotyl elongation by providing robustness to the rhythmic growth pattern. Our data demonstrate that BIN2 redundantly regulates nuclear accumulation of BES1/ BZR1 and the stability of PIF4, coactivator function of these factors thereby serving as a highly sensitive and robust mechanism for external cue integration, enabling the plant to adapt its growth and development to environmental changes.

\section{Materials and methods}

\section{Plant materials and growth conditions}

All of the plants used in this study were in the Col-0 ecotype. To generate the pPIF4: :PIF4-HA pif4pif5 transgenic lines, a geno- 
mic fragment, including a $2.4-\mathrm{kb}$ promoter region and the fulllength PIF4 coding sequence without stop codon, was amplified with the PIF4promoter-f and PIF4_YFPr primers and cloned into the pENTRY S/D-TOPO (Invitrogen) vector. The pPIF4: PIF41A construct was obtained by substituting the NcoI-AgeI fragment of the pPIF4: :PIF4 TOPO plasmid with the corresponding fragment, including the PIF41A mutation. These genomic regions were mobilized by LR clonase into the pGWB13-pPZP destination vector and transformed via Agrobacterium into the pif 4 pif5 mutant. bes1-D PIF41A plants were generated by crossing the pPIF4: :PIF41A-HA plants into the bes1-D pif4pif5 mutants.

Seeds were transferred to vertical MS plates supplemented with the different chemical treatments and grown in darkness or continuous red light for 5-7 d, as specified. Plates were photographed, and the Image J software was used to measure the seedlings' hypocotyl length. Diurnal growth and protein accumulation studies were performed in plants grown in short days (8 h light/16 h dark).

\section{Protein interaction assays}

The full-length BIN2, PIF4, and BES1 coding regions were cloned into the pENTRY S/D-TOPO vector for recombination into the yeast and plant expression vectors. Yeast two-hybrid assays were performed with the GATEWAY-modified pGBKT7 and pGADT7 vectors (Clontech). Both bait and prey constructs were transformed into AH109 cells and selected on SD-LTHA medium. For pull-down assays, ${ }^{35}$ S-Met-radiolabeled PIF4 and BES proteins were synthesized with the TNT T7-coupled reticulocyte lysate system (Promega) and incubated with glutathione-Sepharose beads (GE Healthcare) with the bound GST and GST-BIN2 proteins. For co-IP studies, extracts of $N$. benthamiana leaves expressing the BIN2-HA and PIF4GFP proteins were incubated with anti-HA magnetic beads ( $\mu$ MACS epitope tag, Miltenyi Biotec), and $15 \mu \mathrm{L}$ (anti-HA) and $35 \mu \mathrm{L}$ (anti-GFP) of the immunoprecipitated fraction were used for detection of the proteins. The BIN2 and PIF4 coding regions were fused to the $\mathrm{N}$-terminal and $\mathrm{C}$-terminal YFP fragments of the YFN43 and YFC43 vectors and coinfiltrated into $N$. benthamiana leaves. Leaves were observed $2 \mathrm{~d}$ after infiltration.

\section{In vitro kinase and phosphorylation assays}

PIF4-His, PIF41A-His, MBP-BES1, and BIN2-GST fusion proteins were purified using glutathione agarose (Clontech), amylose agarose (New England Biolabs), or Ni-NTA agarose (Qiagen) beads. For in vitro kinase assays, PIF4-His, MBP-BES1, and GST-BIN2 proteins were coincubated with ${ }^{32} \mathrm{P} \gamma$-ATP for 40 min at $37^{\circ} \mathrm{C}$. Two micromolar and $20 \mu \mathrm{M}$ of the BIN2 kinase inhibitor bikinin (Calbiochem) were added to the incubation mix for specific inhibition. Extracts of mock-, BL-, and BRZtreated seedlings were incubated for $1 \mathrm{~h}$ at $37^{\circ} \mathrm{C}$ with or without CIP (New England Biolabs) to assay for in vivo protein phosphorylation.

\section{qRT-PCR gene expression analysis}

Total RNA was extracted by using the high pure RNA isolation kit (Roche). The SuperScript II reverse transcriptase (Invitrogen) was used for cDNA synthesis, and quantitative real-time RTPCR amplification wasperformed in the 7500 real-time PCR system (Applied Biosystems), following the manufacture's recommendations. PP2A was used as an internal control. Genespecific primers are listed in Supplemental Table 1.

\section{Confocal microscopy}

GFP fluorescence of the split YFP in BiFC-infiltrated $N$. benthamiana leaves or in roots of the transgenic 35S: :PIF4-GFP and 35S: :PIF41A-GFP lines was imaged using an inverted Leica TCS SP5 spectral confocal microscope. Fluorescence was excited with a 488-nm ion argon laser, and emission images were collected in the 500 - to $600-\mathrm{nm}$ range.

\section{Acknowledgments}

We are indebted to Tai-ping Sun, Peter Hedden, Yanhai Yin, and Ana Caño for providing us the $p R G A:$ GFP-RGA and ga1-3 mutant lines in the Col-0 background and for bin2-1 and bes1$d$ mutant seeds. We thank Claus Schwechheimer for the GID1aGFP transgenic lines and the generous gift of the anti-RGA antibody, Miguel Blázquez and David Alabadí for the diurnal growth measurements and fruitful discussions on the work, and Enrique Rojo for his invaluable help with the confocal microscopy. S.B.-G. is recipient of a FPI predoctoral grant, and C.M. holds a post-doctoral reincorporation contract from the Spanish Ministry of Science and Innovation. M.d.L. was awarded a FPI predoctoral grant, and J.-M.D was awarded an I3P post-doctoral contract from the Spanish MICIIN. This work was supported by grants BIO2008-04160, BIO2011-30546, and CSD2007-00057 from the Spanish Ministry of Science and Innovation.

\section{References}

Achard P, Liao L, Caifu C, Desnos T, Bartlett J, Fu X, Harberd N. 2007. DELLAs contribute to plant photomorphogenesis. Plant Physiol 143: 1163-1172.

Alabadí D, Gil J, Blázquez MA, García-Martínez JL. 2004. Gibberellins repress photomorphogenesis in darkness. Plant Physiol 134: 1050-1057.

Bai MY, Shang JX, Oh E, Bai Y, Zentella R, Sun TP, Wang ZY. 2012. Brassinosteroid, gibberellin and phytochrome impinge on a common transcription module in Arabidopsis. Nat Cell Biol 14: 810-817.

Bauer D, Viczián A, Kircher S, Nobis T, Nitschke R, Kunkel T, Panigrahi KC, Adám E, Fejes E, Schäfer E, et al. 2004. Constitutive photomorphogenesis 1 and multiple photoreceptors control degradation of phytochrome interacting factor 3, a transcription factor required for light signaling in Arabidopsis. Plant Cell 16: 1433-1445.

Castillon A, Shen H, Huq E. 2007. Phytochrome interacting factors: central players in phytochrome-mediated light signaling networks. Trends Plant Sci 12: 514-521.

Clouse SD. 2011. Brassinosteroid signal transduction: from receptor kinase activation to transcriptional networks regulating plant development. Plant Cell 23: 1219-1230.

De Lucas M, Davière JM, Rodríguez-Falcón M, Pontin M, IglesiasPedraz JM, Lorrain S, Fankhauser C, Blázquez MA, Titarenko E, Prat S. 2008. A molecular framework for light and gibberellin control of cell elongation. Nature 451: 480-484.

De Rybel B, Audenaert D, Vert G, Rozhon W, Mayerhofer J, Peelman F, Coutuer S, Denayer T, Jansen L. 2009. Chemical inhibition of a subset of Arabidopsis thaliana GSK3-like kinases activates brassinosteroid signaling. Chem Biol 16: 594-604.

Dill A, Thomas SG, Hu J, Steber CM, Sun TP. 2004. The Arabidopsis F-box protein SLEEPY1 targets gibberellin signaling repressors for gibberellin-induced degradation. Plant Cell 16: 1392-1405.

Feng S, Martinez C, Gusmaroli G, Wang Y, Zhou J, Wang F, Chen L, Yu L, Iglesias-Pedraz JM, Kircher S, et al. 2008. 
Coordinated regulation of Arabidopsis thaliana development by light and gibberellins. Nature 451: 475-479.

Fleet CM, Sun TP. 2004. A DELLAcate balance: the role of gibberellin in plant morphogenesis. Curr Opin Plant Biol 8: 77-85.

Fu X, Richards DE, Fleck B, Xie D, Burton N, Harberd NP. 2004. The Arabidopsis mutant sleepylgar2-1 protein promotes plant growth by increasing the affinity of the SCF ${ }^{\mathrm{SLY} 1} \mathrm{E} 3$ ubiquitin ligase for DELLA protein substrates. Plant Cell 16: 1406-1418.

Gallego-Bartolomé J, Minguet EG, Marín JA, Prat S, Blázquez MA, Alabadí D. 2010. Transcriptional diversification and functional conservation between DELLA proteins in Arabidopsis. Mol Biol Evol 27: 1247-1256.

Gallego-Bartolomé J, Minguet EG, Grau-Enguix F, Abbas M, Locascio A, Thomas SG, Alabadí D, Blázquez MA. 2012. Molecular mechanism for the interaction between gibberellin and brassinosteroid signaling pathways in Arabidopsis. Proc Natl Acad Sci 109: 13446-13451.

Gampala S, Kim TW, He JX, Tang W, Deng Z, Bai M, Guan S, Lalonde S, Sun Y, Gendron JM, et al. 2007. An essential role for 14-3-3 proteins in brassinosteroid signal transduction in Arabidopsis. Dev Cell 13: 177-189.

Goda H, Sawa S, Asami T, Fujioka S, Shimada Y, Yoshida S. 2004. Comprehensive comparison of auxin-regulated and brassinosteroid-regulated genes in Arabidopsis. Plant Physiol 134: $1555-1573$.

Goda H, Sasaki E, Akiyama K, Maruyama-Nakashita A, Nakabayashi K, Li W, Ogawa M, Yamauchi Y, Preston J, Aoki K, et al. 2008. The AtGenExpress hormone and chemical treatment data set: experimental design, data evaluation, model data analysis and data access. Plant $J$ 55: 526-542.

He JX, Gendron JM, Yang Y, Li J, Wang ZY. 2002. The GSK3-like kinase BIN2 phosphorylates and destabilizes BZR1, a positive regulator of the brassinosteroid signaling pathway in Arabidopsis. Proc Natl Acad Sci 99: 10185-10190.

He JX, Gendron JM, Sun Y, Gampala SS, Gendron N, Sun CQ, Wang ZY. 2005. BZR1 is a transcriptional repressor with dual roles in brassinosteroid homeostasis and growth responses. Science 307: 1634-1638.

Hornitschek P, Kohnen MV, Lorrain S, Rougemont J, Ljung K, Lopez-Vidriero I, Franco-Zorrilla JM, Solano R, Trevisan M, Pradervan S. 2012. Phytochrome interacting factors 4 and 5 control seedling growth in changing light conditions by directly controlling auxin signaling. Plant $J$ 71: 699-711.

Kim TW, Wang ZY. 2010. Brassinosteroid signal transduction from receptor kinases to transcription factors. Annu Rev Plant Biol 61: 681-704.

Kim TW, Guan S, Sun Y, Deng Z, Tang W, Shang JX, Sun Y, Burlingame AL, Wang ZY. 2009. Brassinosteroid signal transduction from cell-surface receptor kinases to nuclear transcription factors. Nat Cell Biol 11: 1254-1260.

Kinoshita T, Cano-Delgado A, Seto H, Hiranuma S, Fujioka S, Yoshida S, Chory J. 2005. Binding of brassinosteroids to the extracellular domain of plant receptor kinase BRI1. Nature 433: $167-171$.

Leivar P, Quail PH. 2011. PIFs: pivotal components in a cellular signaling hub. Trends Plant Sci 16: 19-28.

Leivar P, Monte E, Oka Y, Liu T, Carle C, Castillon A, Huq E, Quail PH. 2008. Multiple phytochrome-interacting bHLH transcription factors repress premature seedling photomorphogenesis in darkness. Curr Biol 18: 18151823.

Leivar P, Tepperman JM, Monte E, Calderon RH, Liu TL, Quail PH. 2009. Definition of early transcriptional circuitry involved in light-induced reversal of PIF-imposed repression of photomorphogenesis in young Arabidopsis seedlings. Plant Cell 21: 3535-3553.

Li J, Nam KH. 2002. Regulation of brassinosteroid signaling by a GSK3/SHAGGY-like kinase. Science 295: 1299-1301.

Li J, Nagpal P, Vitart V, McMorris TC, Chory J. 1996. A role for brassinosteroids in light-dependent development of Arabidopsis. Science 272: 398-401.

Li QF, Wang C, Jiang L, Li S, Sun SSM, He JX. 2012. An interaction between BZR1 and DELLAs mediates direct signaling crosstalk between brassinosteroids and gibberellins in Arabidopsis. Sci Signal 5: ra72.

Lorrain S, Allen T, Duek PD, Whitelam GC, Fankhauser C. 2008. Phytochrome-mediated inhibition of shade avoidance involves degradation of growth-promoting bHLH transcription factors. Plant J 53: 312-323.

Luo XM, Lin WH, Zhu S, Zhu JY, Sun Y, Fan XY, Cheng M, Hao Y, Oh E, Tian $M$, et al. 2010. Integration of light and brassinosteroid signaling pathways by a GATA transcription factor in Arabidopsis. Dev Cell 19: 872-883.

Michael TP, Breton G, Hazen SP, Priest H, Mockler TC, Kay SA, Chory J. 2008. A morning-specific phytohormone gene expression program underlying rhythmic plant growth. PLoS Biol 6: e225.

Mora-Garcia S, Vert G, Yin Y, Cano-Delgado A, Cheong H, Chory J. 2004. Nuclear protein phosphatases with Kelchrepeat domains modulate the response to brassinosteroids in Arabidopsis. Genes Dev 18: 448-460.

Murase K, Hirano Y, Sun TP, Hakoshima T. 2008. Gibberellininduced DELLA recognition by the gibberellin receptor GID1. Nature 456: 459-463.

Nam KH, Li J. 2002. BRI1/BAK1, a receptor kinase pair mediating brassinosteroid signaling. Cell 110: 203-212.

Nagata N, Min YK, Nakano T, Asami T, Yoshida S. 2000. Treatment of dark-grown Arabidopsis thaliana with a brassinosteroidbiosynthesis inhibitor, brassinazole, induces some characteristics of light-grown plants. Planta 211: 781-790.

Nemhauser JL, Chory J. 2004. BRing it on: new insights into the mechanism of brassinosteroid action. J Exp Bot 55: 265-270.

Nemhauser JL, Mockler TC, Chory J. 2004. Interdependency of brassinosteroid and auxin signaling in Arabidopsis. PLoS Biol 2: e258.

Nemhauser JL, Hong F, Chory J. 2006. Different plant hormones regulate similar processes through largely nonoverlapping transcriptional responses. Cell 126: 467-475.

Ni W, Xu SL, Chalkley RJ, Pham TN, Guan S, Maltby DA, Burlingame AL, Wang ZY, Quail PH. 2013. Multisite lightinduced phosphorylation of the transcription factor PIF3 is necessary for both its rapid degradation and concomitant negative feedback modulation of photoreceptor phyB levels in Arabidopsis. Plant Cell 25: 2679-2698.

Nozue K, Maloof JN. 2006. Diurnal regulation of plant growth. Plant Cell Environ 29: 396-408.

Nozue K, Covington MF, Duek PD, Lorrain S, Fankhauser C, Harmer SL, Maloof JN. 2007. Rhythmic growth explained by coincidence between internal and external cues. Nature 448: 358-361.

Nusinow DA, Helfer A, Hamilton EE, King IJ, Imaizumi T, Schultz TF, Farré EM, Kay SA. 2011. The ELF4-ELF3-LUX complex links the circadian clock to diurnal control of hypocotyl growth. Nature 475: 398-402.

Oh E, Kang H, Yamaguchi S, Park J, Lee D, Kamiya Y, Choi G. 2009. Genome-wide analysis of genes targeted by PHYTOCHROME INTERACTING FACTOR 3-LIKE5 during seed germination in Arabidopsis. Plant Cell 21: 403419. 
Oh E, Zhu JY, Wang ZY. 2012. Interaction between BZR1 and PIF4 integrates brassinosteroid and environmental responses. Nat Cell Biol 14: 802-809.

Peng P, Yan Z, Zhu Y, Li J. 2008. Regulation of the Arabidopsis GSK3-like kinase BRASSINOSTEROID-INSENSITIVE 2 through proteasome-mediated protein degradation. Mol Plant 1: 338-346.

Peng P, Zhao J, Zhu Y, Asami T, Li J. 2010. A direct docking mechanism for a plant GSK3-like kinase to phosphorylate its substrates. I Biol Chem 285: 24646-24653.

Quail PH. 2010. Phytochromes. Curr Biol 20: R504-R507.

Ryu H, Cho H, Kim K, Hwang I. 2010. Phosphorylation dependent nucleocytoplasmic shuttling of BES1 is a key regulatory event in brassinosteroid signaling. Mol Cells 29: 283-290.

Santiago J, Henzler C, Hothorn M. 2013. Molecular mechanism for plant steroid receptor activation by somatic embryogenesis co-receptor kinases. Science 341: 889-892.

She J, Han Z, Kim TW, Wang J, Cheng W, Chang J, Shi S, Wang J, Yang M, Wang ZY, et al. 2011. Structural insight into brassinosteroid perception by BRI1. Nature 474: 472-476.

Shen Y, Khanna R, Carle CM, Quail PH. 2007. Phytochrome induces rapid PIF5 phosphorylation and degradation in response to red-light activation. Plant Physiol 145: 1043-1051.

Shimada A, Ueguchi-Tanaka M, Nakatsu T, Nakajima M, Naoe Y, Ohmiya H, Kato H, Matsuoka M. 2008. Structural basis for gibberellin recognition by its receptor GID1. Nature 456: 520-523.

Steber CM, McCourt P. 2001. A role for brassinosteroids in germination in Arabidopsis. Plant Physiol 125: 763-769.

Sun Y, Fan XY, Cao DM, Tang W, He K, Zhu JY, He JX, Bai MY, Zhu S, Oh E, et al. 2010. Integration of brassinosteroid signal transduction with the transcription network for plant growth regulation in Arabidopsis. Dev Cell 19: 765-777.

Sun J, Qi L, Li Y, Chu J, Li C. 2012. PIF4-mediated activation of YUCCA8 expression integrates temperature into the Auxin pathway in regulating Arabidopsis hypocotyl growth. PLoS Genet 8: e1002594.

Tanaka K, Nakamura Y, Asami T, Yoshida S, Matsuo T, Okamoto S. 2003. Physiological roles of brassinosteroids in early growth of Arabidopsis: brassinosteroids have a synergistic relationship with gibberellin as well as auxin in light-grown hypocotyl elongation. J Plant Growth Regul 22: 259-271.

Tang W, Yuan M, Wang R, Yang Y, Wang C, Oses-Prieto JA, Kim TW, Zhou HW, Deng Z, Gampala SS, et al. 2011. PP2A activates brassinosteroid-responsive gene expression and plant growth by dephosphorylating BZR1. Nat Cell Biol 13: 124-131.

Vert G, Chory J. 2006. Downstream nuclear events in brassinosteroid signalling. Nature 441: 96-100.

Vert G, Nemhauser JL, Geldner N, Hong F, Chory J. 2005. Molecular mechanisms of steroid hormone signaling in plants. Annu Rev Cell Dev Biol 21: 177-201.

Wang ZY, Nakano T, Gendron J, He J, Chen M, Vafeados D, Yang Y, Fujioka S, Yoshida S, Asami T, et al. 2002. Nuclearlocalized BZR1 mediates brassinosteroid-induced growth and feedback suppression of brassinosteroid biosynthesis. Dev Cell 2: 505-513.

Wang X, Kota U, He K, Blackburn K, Li J, Goshe MB, Huber SC, Clouse SD. 2008. Sequential transphosphorylation of the BRI1/BAK1 receptor kinase complex impacts early events in brassinosteroid signaling. Dev Cell 15: 220-235.

Wang ZY, Bai MY, Oh E, Zhu JY. 2012. Brassinosteroid signaling network and regulation of photomorphogenesis. Annu Rev Genet 46: 701-724.

Willige BC, Chosh S, Nill C, Zourelidou M, Dohmann E, Maier A, Schwechheimer C. 2007. The DELLA domain of GA INSENSITIVE mediates the interaction with the GA
INSENSITIVE DWARF1A gibberellin receptor of Arabidopsis. Plant Cell 19: 1209-1220.

Yamashino T, Nomoto Y, Lorrain S, Miyachi M, Ito S, Nakamichi N, Fankhauser C, Mizuno T. 2013. Verification at the protein level of the PIF4-mediated external coincidence model for the temperature-adaptive photoperiodic control of plant growth in Arabidopsis thaliana. Plant Signal Behav 8: e23390.

Yin Y, Wang ZY, Mora-Garcia S, Li J, Yoshida S, Asami T, Chory J. 2002. BES1 accumulates in the nucleus in response to brassinosteroids to regulate gene expression and promote stem elongation. Cell 109: 181-191.

Yin Y, Vafeados D, Tao Y, Yoshida S, Asami T, Chory J. 2005. A new class of transcription factors mediates brassinosteroidregulated gene expression in Arabidopsis. Cell 120: 249-259.

Yu X, Li L, Zola J, Aluru M, Ye H, Foudree A, Guo H, Anderson S, Aluru S, Liu P, et al. 2011. A brassinosteroid transcriptional network revealed by genome-wide identification of BES1 target genes in Arabidopsis thaliana. Plant J 65: 634-646.

Zhao J, Peng P, Schmitz RJ, Decker AD, Tax FE, Li J. 2002. Two putative BIN2 substrates are nuclear components of brassinosteroid signaling. Plant Physiol 130: 1221-1229.

Zhang Y, Mayba O, Pfeiffer A, Shi H, Tepperman JM, Speed TP, Quail PH. 2013. A quartet of PIF bHLH factors provides a transcriptionally centered signaling hub that regulates seedling morphogenesis through differential expression-patterning of shared target genes in Arabidopsis. PLoS Genet 9: e1003244. 


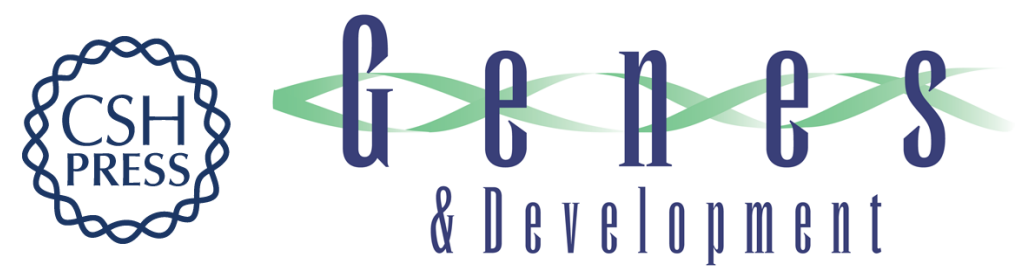

\section{BR-dependent phosphorylation modulates PIF4 transcriptional activity and shapes diurnal hypocotyl growth}

Stella Bernardo-García, Miguel de Lucas, Cristina Martínez, et al.

Genes Dev. 2014, 28:

Access the most recent version at doi:10.1101/gad.243675.114

\section{Supplemental http://genesdev.cshlp.org/content/suppl/2014/07/31/28.15.1681.DC1 \\ Material}

References This article cites 70 articles, 23 of which can be accessed free at:

http://genesdev.cshlp.org/content/28/15/1681.full.html\#ref-list-1

Creative This article is distributed exclusively by Cold Spring Harbor Laboratory Press for the first

Commons six months after the full-issue publication date (see

License http://genesdev.cshlp.org/site/misc/terms.xhtml). After six months, it is available under a Creative Commons License (Attribution-NonCommercial 4.0 International), as described at http://creativecommons.org/licenses/by-nc/4.0/.

Email Alerting Receive free email alerts when new articles cite this article - sign up in the box at the top Service right corner of the article or click here.

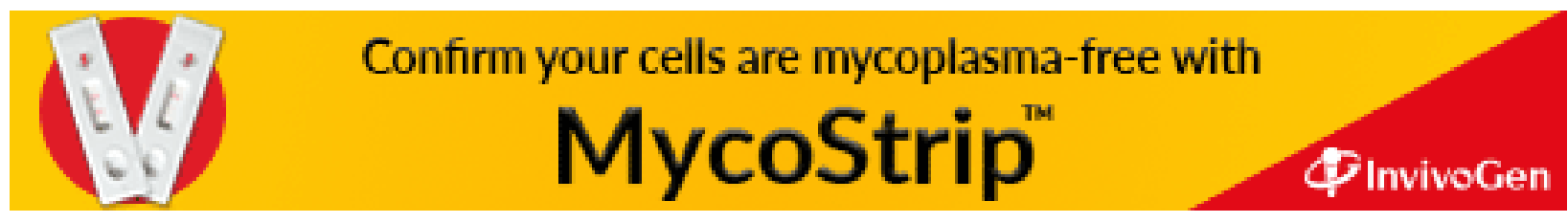

